\title{
Light Mesons in Photoproduction
}

\author{
Anna Kropivnitskaya (on behalf of the H1 Collaboration) \\ Institute for Theoretical and Experimental Physics \\ Bolshaya Cheremushkinskaya 25, Moscow - Russia
}

\begin{abstract}
Inclusive non-diffractive photoproduction of $\rho(770)^{0}, K^{*}(892)^{0}$ and $\phi(1020)$ mesons is investigated with the $\mathrm{H} 1$ detector in $e p$ collisions at HERA. The corresponding average $\gamma p$ centre-of-mass energy is $210 \mathrm{GeV}$. The mesons are measured in the transverse momentum range $0.5<p_{T}<7 \mathrm{GeV}$ and the rapidity range $\left|y_{\text {lab }}\right|<1$. Differential cross sections are presented as a function of transverse momentum and rapidity, and are compared to the predictions of hadroproduction models.
\end{abstract}

\section{Introduction}

High energy particle collisions which give rise to large multiplicities of produced hadrons provide an opportunity to study the hadronisation process, in which quarks and gluons convert to colourless hadrons. Since most hadrons are produced with low transverse momenta, the theory of perturbative quantum chromodynamics (pQCD) is not applicable to describe hadronisation. The production of long-lived hadrons and resonances at high energies was studied in detail in electron-positron $\left(e^{-} e^{+}\right)$collisions at LEP using $Z^{0}$ decays [2]. Measurements in high energy hadronic interactions have so far been restricted to long-lived hadrons and hadrons containing heavy quarks. Recently, the production of the hadronic resonances $\rho(770)^{0}, K^{*}(892)^{0}$ and $\phi(1020)$ was measured in heavy-ion and proton-proton $(p p)$ collisions at RHIC [3]. The electron-proton $(e p)$ collider HERA allows the study of particle production in quasi-real photon-proton $(\gamma p)$ collisions, where the nuclear density is much lower than at RHIC. This is particularly interesting, because the $\gamma p$ centre-of-mass energy at HERA is about the same as for colliding nucleons at RHIC.

\section{Phenomenology}

The invariant differential cross section for meson production can be expressed as a function of the meson's transverse momentum $p_{T}$ and its rapidity $y_{l a b}$, assuming azimuthal symmetry. Hadrons produced in hadronic collisions are approximately uniformly distributed in the central rapidity range, while their transverse momentum spectra fall steeply with increasing $p_{T}$. It is convenient to parametrise the invariant differential cross section of the produced hadrons with a power law distribution,

$$
\frac{1}{\pi} \frac{d^{2} \sigma^{\gamma p}}{d p_{T}^{2} d y_{l a b}}=\frac{A}{\left(E_{T_{0}}+E_{T}^{k i n}\right)^{n}}
$$

where $E_{T}^{k i n}=\sqrt{m_{0}^{2}+p_{T}^{2}}-m_{0}$ is the transverse kinetic energy, $m_{0}$ is the nominal resonance mass, $A$ is a normalisation factor independent of $p_{T}$ and $E_{T_{0}}$ a free parameter. When $E_{T}^{k i n} \lesssim$ $E_{T_{0}}$, the power law function (1) behaves like a Boltzmann distribution $\exp \left(-E_{T}^{k i n} / T\right)$, with $T=E_{T_{0}} / n$. This exponential behaviour of hadronic spectra follows from a thermodynamic model of hadroproduction [4]. In this framework, the parameter $T$ plays the role of the 
temperature at which hadronisation takes place. At high $E_{T}^{k i n}$, the power law originates from a convolution of the parton densities of the colliding particles with the cross sections of parton-parton interactions. Assuming that the $p_{T}$ and $y_{l a b}$ dependencies factorise, the normalisation coefficient $A$ is related to the single differential cross section $d \sigma / d y_{\text {lab }}$ obtained after integrating equation (1) over $p_{T}^{2}$ :

$$
A=\frac{d \sigma}{d y_{l a b}} \frac{(n-1)(n-2)\left(E_{T_{0}}\right)^{n-1}}{2 \pi\left(E_{T_{0}}+(n-2) m_{0}\right)} .
$$

\section{Inclusive Photoproduction of $\rho^{0}(770), K^{*}(892)^{0}$ and $\phi(1020)$ Mesons}

First measurements of inclusive non-diffractive photoproduction of $\rho^{0}, K^{* 0}$ and $\phi$ mesons at HERA are presented by the H1 collaboration [5]. Figure 1 shows clear signals of the $\rho^{0}(a$ and $b), K^{* 0}(c)$ and $\phi(d)$ mesons. To extract these signals, the distributions of the respective invariant masses of their decay products, $m_{\pi^{+} \pi^{-}}, m_{K^{ \pm} \pi^{\mp}}$ and $m_{K^{+} K^{-}}$, are fitted using a function composed of three parts:

$F(m)=B(m)+\sum R(m)+\sum S(m)$.

The terms correspond to contributions from the combinatorial background, $B(m)$, from hidden resonance effects, termed reflections, $R(m)$, and from the relevant signals, $S(m)$, respectively. The function $S(m)$ is a convolution of a relativistic Breit-Wigner function and a detector resolution function.
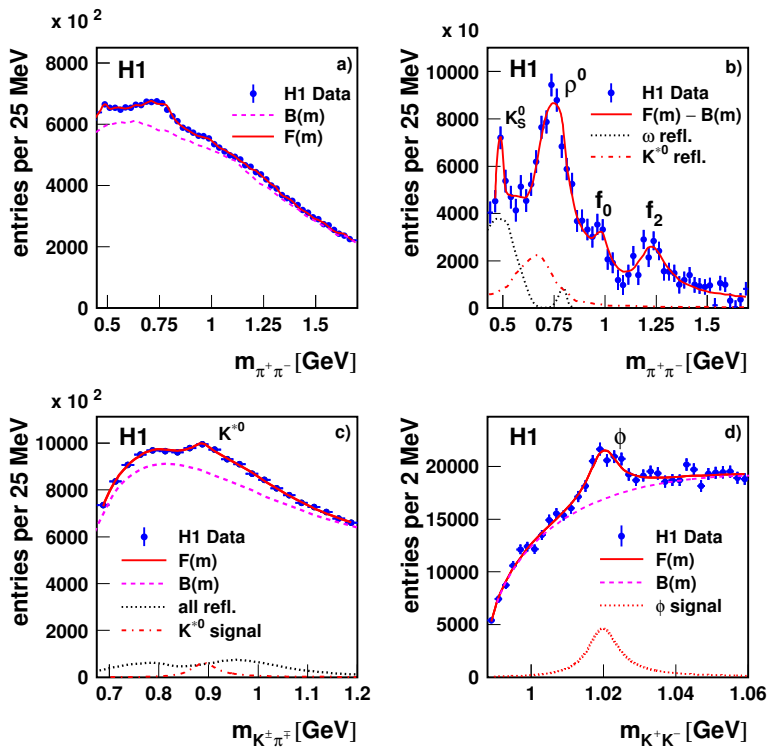

Figure 1: The invariant mass spectra for $\pi^{+} \pi^{-},(a, b)$, $K^{ \pm} \pi^{\mp},(c)$, and $K^{+} K^{-},(d)$, pairs with the result of the fit. In $b)$, the data and the fit $F(m)$ are shown after subtraction of the combinatorial background $B(m)$.

For the $\rho^{0}$ meson, the detector resolution is significantly smaller than its width. However, Bose-Einstein correlations (BEC) between the $\rho^{0}$ decay pions and other pions in the event strongly distort the $\rho^{0}$ line shape. The BEC play an important role in broadening the $\rho^{0}$ mass peak and in shifting it towards lower masses. Similar effects are observed in $p p$ and heavy-ion collisions at RHIC [3] and in $e^{+} e^{-}$collisions at LEP using $Z^{0}$ decays [2]. It is therefore important to check that the Monte Carlo model used for the extraction of the cross sections describes the di-pion spectra in the data. The data spectrum and the Monte Carlo simulations with and without BEC are shown in Figure 2. The Monte Carlo model with $\mathrm{BEC}$ is in good agreement with the data in the region of the $\rho^{0}$ resonance, whereas the model without BEC fails to describe the di-pion mass spectrum. 
The inclusive non-diffractive photoproduction cross sections for $\rho^{0}, K^{* 0}$ and $\phi(1020)$ mesons in the kinematic region $Q^{2}<0.01 \mathrm{GeV}^{2}$, $174<W<256 \mathrm{GeV}$ and for $p_{T}>0.5 \mathrm{GeV}$ and $\left|y_{\text {lab }}\right|<1$ are found to be:

$$
\begin{aligned}
& \sigma_{v i s}^{\gamma p}\left(\gamma p \rightarrow \rho^{0} X\right)=25600 \pm 1800 \pm 2700 \mathrm{nb} \\
& \sigma_{v i s}^{\gamma p}\left(\gamma p \rightarrow K^{* 0} X\right)=6260 \pm 350 \pm 860 \mathrm{nb} ; \\
& \sigma_{v i s}^{\gamma p}(\gamma p \rightarrow \phi X)=2400 \pm 180 \pm 340 \mathrm{nb} .
\end{aligned}
$$

The first error is statistical and the second systematic. Note that the $K^{* 0}$ cross section is the sum of the particle and antiparticle contributions divided by 2 .

The differential cross sections for the photoproduction of $\rho^{0}, K^{* 0}$, and $\phi$ mesons are presented in Figure 3 . Within the rapidity range of this measurement, the resonance production rates are constant as a function of rapidity, within errors. The transverse momentum spectra of the $\rho^{0}, K^{* 0}$ and $\phi$ mesons can be parametrised by function (1), where $d \sigma / d y_{l a b}$ in equation (2) corresponds to the average value of the cross section over central rapidities, $\left\langle d \sigma / d y_{l a b}\right\rangle_{\left|y_{l a b}\right|<1}$. In the fit, the value of the power $n$ is fixed to be 6.7, as derived previously from measurements of charged particle spectra by the $\mathrm{H} 1$ collaboration [6] which gave $n=6.7 \pm 0.3$. The power law distribution, with this value of $n$, describes $K_{S}^{0}$ meson, $\Lambda^{0}$ baryon and $D^{* \pm}$ meson production [7] at HERA, as is shown in Figure 4. The results of fits of function (1) to the data are shown in Figure 3a). In Table 1, the parameters of the fit and the average transverse kinetic energy $\left\langle E_{T}^{k i n}\right\rangle$, the average transverse energy $\left\langle E_{T}\right\rangle=\left\langle E_{T}^{k i n}\right\rangle+m_{0}$ and the average transverse momentum $\left\langle p_{T}\right\rangle=\sqrt{\left\langle E_{T}\right\rangle^{2}-m_{0}^{2}}$ derived from (1) are presented. The errors include the experimental uncertainty on the value of $n$. Also given are the $\left\langle p_{T}\right\rangle$ value measured at $\mathrm{RHIC}$ in $p p$ and $\mathrm{Au}-\mathrm{Au}$ collisions.

It is interesting to observe that the resonances with different masses, lifetimes and strangeness content are produced with about the same value of the average transverse kinetic energy $\left\langle E_{T}^{k i n}\right\rangle$. This observation supports the thermodynamic picture of hadronic interactions [4], in which the primary hadrons are thermalised during the interaction. The values

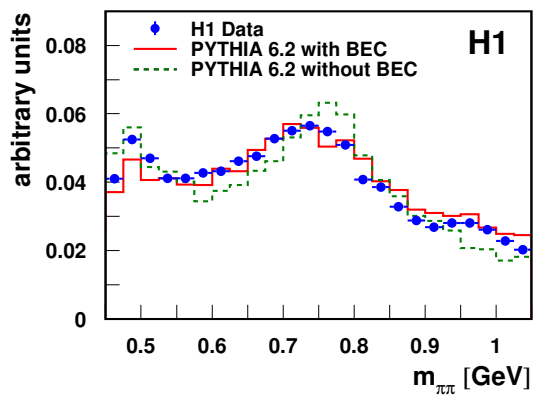

Figure 2: The unlike-sign di-pion invariant mass spectrum after subtracting the like-sign contribution, normalised to the total number of entries.
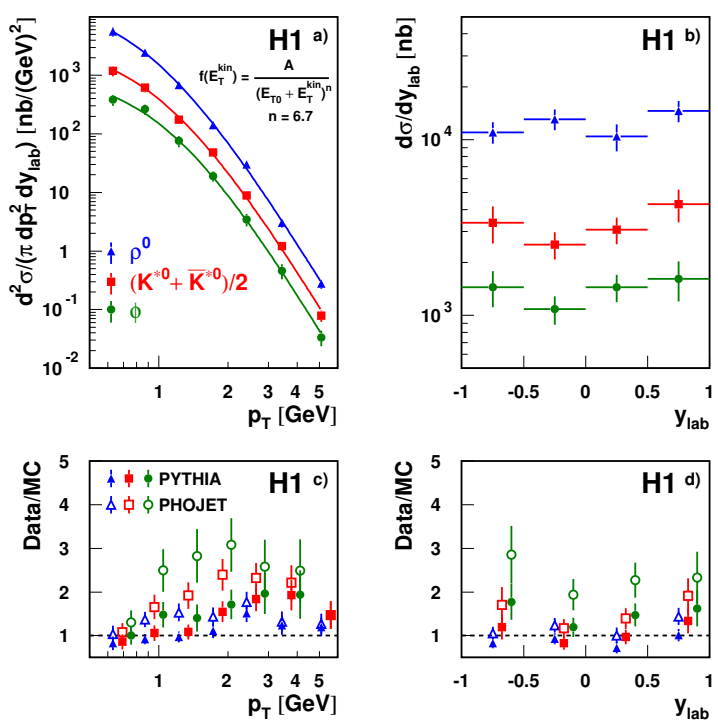

Figure 3: The measured differential non-diffractive photoproduction cross-sections for $\rho^{0}, K^{* 0}$ and $\phi$ mesons. (For more details see the text.) 
of $\left\langle p_{T}\right\rangle$ for $\rho^{0}, K^{* 0}$ and $\phi$ mesons are similar in $\gamma p$ and $p p$ collisions with about the same centre-of-mass energy $\sqrt{s} \approx 200 \mathrm{GeV}$, while these values are all higher in $\mathrm{Au}$-Au collisions.

The PYTHIA and PHOJET models do not describe the shape of the measured $p_{T}$ spectra. These observations are illustrated in Figures 3c) and 3d). Moreover, contrary to the data, the Monte Carlo $p_{T}$ spectra are not described by the power law function (1).

The measurements in the visible kinematic range of the $\rho^{0}, K^{* 0}$ and $\phi$ mesons, $p_{T}>0.5 \mathrm{GeV}$ and $\left|y_{l a b}\right|<1$, are extrapolated to the full $p_{T}$ range using the parametrisation (1) to determine the total inclusive non-diffractive photoproduction cross sections. The extrapolation factors are of order two. To calculate the ratios of the $\phi$ and $K^{* 0}$ cross sections, their average values of the differential cross sections $\left\langle d \sigma / d y_{l a b}\right\rangle_{\left|y_{l a b}\right|<1}$ are used. In Figure 5, $R\left(\phi / K^{* 0}\right)$ is compared to the corresponding ratios measured by STAR in $p p$ and $\mathrm{Au}-\mathrm{Au}$ collisions [3] at $\sqrt{s_{N N}}=200 \mathrm{GeV}$. Although the rapidity ranges at the $\mathrm{H} 1$ and STAR experiment differ ${ }^{\mathrm{a}}$, the resulting ratios for $p p$ and $\gamma p$ interactions are very close. For $\phi$ meson production a tendency to be more abundant in $\mathrm{Au}-\mathrm{Au}$ collisions is observed.

\begin{tabular}{|l|r|c|c|c|}
\hline & & $\rho^{0}$ & $\left(K^{* 0}+\bar{K}^{* 0}\right) / 2$ & $\phi$ \\
\hline \multirow{4}{*}{$\gamma p$} & $\left\langle d \sigma / d y_{\text {lab }}\right\rangle_{\left|y_{\text {lab }}\right|<1}[\mathrm{nb}]$ & $23600 \pm 2700$ & $5220 \pm 600$ & $1850 \pm 230$ \\
\cline { 2 - 5 } & $T[\mathrm{GeV}]$ & $0.151 \pm 0.011$ & $0.166 \pm 0.012$ & $0.170 \pm 0.012$ \\
\cline { 2 - 5 } & $\left\langle E_{T}\right\rangle[\mathrm{GeV}]$ & $1.062 \pm 0.018$ & $1.205 \pm 0.020$ & $1.333 \pm 0.022$ \\
\cline { 2 - 5 } & $\left\langle E_{T}^{k i n}\right\rangle[\mathrm{GeV}]$ & $0.287 \pm 0.018$ & $0.313 \pm 0.020$ & $0.314 \pm 0.022$ \\
\cline { 2 - 5 } & $\left\langle p_{T}\right\rangle[\mathrm{GeV}]$ & $0.726 \pm 0.027$ & $0.810 \pm 0.030$ & $0.860 \pm 0.035$ \\
\hline \hline$p p$ & $\left\langle p_{T}\right\rangle_{p p}[\mathrm{GeV}]$ & $0.616 \pm 0.062$ & $0.81 \pm 0.14$ & $0.82 \pm 0.03$ \\
\hline $\mathrm{Au}-\mathrm{Au}$ & $\left\langle p_{T}\right\rangle_{A u A u}[\mathrm{GeV}]$ & $0.83 \pm 0.10$ & $1.08 \pm 0.14$ & $0.97 \pm 0.02$ \\
\hline
\end{tabular}

Table 1: The parameters of function (1) from a fit to the $\rho^{0}, K^{* 0}$ and $\phi$ meson differential cross sections and derived variables are shown. Also provided are results from measurements in $p p$ and $\mathrm{Au}-\mathrm{Au}$ interactions at a nucleon-nucleon centre-of-mass energy $\sqrt{s_{N N}}=200$ $\mathrm{GeV}[3]$ at central rapidities.

\footnotetext{
aThe difference in rapidity between the laboratory frame and the $\gamma p$ frame is about two units at H1.
} 


\section{Conclusions}

First measurements of the inclusive non-diffractive photoproduction of $\rho(770)^{0}, K^{*}(892)^{0}$ and $\phi(1020)$ mesons at HERA are presented. The differential cross sections for the production of these resonances as a function of transverse momentum are described by a power law distribution, while the single differential cross sections as a function

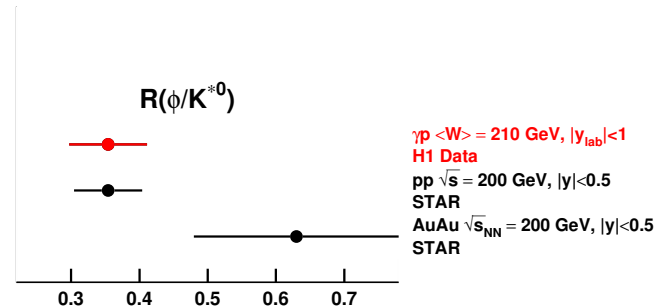
of rapidity are observed to be flat in the visible range. These resonances, despite their different masses, lifetimes and strangeness content are produced with about the same value of the average transverse kinetic energy. This observation supports a thermodynamic picture of hadronic interactions.

The description of the shape of the $\rho^{0}$ resonance produced in $\gamma p$ collisions at HERA is improved by taking Bose-Einstein correlations into account. A similar effect is observed in $p p$ and heavy-ion collisions at RHIC and in $e^{+} e^{-}$annihilation at LEP, using $Z^{0}$ decays.

The cross section ratio $R\left(\phi / K^{* 0}\right)$ is determined and is compared to results obtained in $p p$ and heavy-ion collisions by the STAR experiment at RHIC. The ratio $R\left(\phi / K^{* 0}\right)$ measured in $\gamma p$ interactions is in agreement with the $p p$ results. A tendency for $\phi$ meson production to be more abundant in $\mathrm{Au}-\mathrm{Au}$ collisions is observed.

\section{Acknowledgments}

The support of the Rosnauka, DESY and Helmholtz Association in the framework of the Helmholtz-Russian-Joint-Research Group (HRJRG-002) are gratefully acknowledged. I would like to thank Andrey Rostovtsev for useful discussions.

\section{Bibliography}

\section{References}

[1] Slides: http: //indico. cern. ch/materialDisplay $\cdot$ py? contribId=247\&sessionId=3\&materialld=slides\&conf Id=53294

[2] A. Boehrer, Phys. Rept. 291107 (1997) and the references therein.

[3] STAR Collab., J. Adams, et al., Phys. Rev. Lett. 92092301 (2004); STAR Collab., C. Adler et al., Phys. Rev. C71 064902 (2005); STAR Collab., J. Adams et al., Phys. Lett. B612 181 (2005).

[4] R. Hagedorn, Nuovo Cim. Suppl. 3147 (1965).

[5] H1 Collab., F.D. Aaron et al., Phys. Lett. B673 119 (2009).

[6] H1 Collab., I. Abt et al., Phys. Lett. B328 176 (1994); H1 Collab., C. Adloff et al., Eur. Phys. J. C10 363 (1999).

[7] H1 Collab., C. Adloff et al., Z. Phys. C76 213 (1997); H1 Collab., A. Aktas et al., Eur. Phys. J. C50 251 (2007). 